\title{
The causes and management of nonunion of femoral subtrochanteric shortening osteotomy in a THA patient: a case report
}

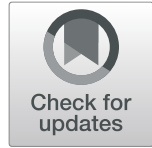

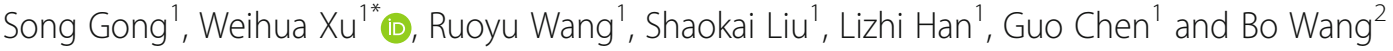

\begin{abstract}
Background: Total hip arthroplasty (THA) is considerably difficult to perform in patients with Crowe type IV developmental dysplasia of the hip (DDH). Some Crowe type IV DDH patients require a femoral subtrochanteric shortening osteotomy to equalize the length of the lower extremities and decrease the difficulty of intraoperative reduction. Subtrochanteric transverse osteotomy has been proven to have superior clinical efficacy, but some cases of nonunion occur.

Case presentation: We present the case of a 62-year-old male patient who underwent right THA with femoral subtrochanteric transverse osteotomy due to Crowe type IV DDH. Nonunion of the osteotomy occurred during the follow-up period. In July 2017, the patient underwent right THA and femoral subtrochanteric transverse osteotomy due to Crowe type IV DDH. In November 2017, a slight feeling of bone rubbing and slight pain in the hip were reported. The ends of the osteotomy had rotated and united poorly. However, the patient requested to undergo continued observation. In December 2017, the patient reported an obvious sensation of bone rubbing and aggravated hip pain. The ends of the osteotomy had rotated and continued to exhibit nonunion. On December 26, 2017, the patient was treated with plate and screw internal fixation with bone morphogenetic protein (BMP) following our suggestion. In August 2018, the ends of the osteotomy had united after internal fixation was applied.

Conclusions: THA with femoral subtrochanteric transverse osteotomy exhibits good efficacy for the treatment of patients with Crowe type IV DDH. However, postoperative nonunion occurs in a small number of cases. The causes of nonunion should be analysed, and effective measures should be taken to prevent this situation. Plate and screw internal fixation with BMP is an effective treatment for nonunion of the ends of an osteotomy.
\end{abstract}

Keywords: Nonunion, Subtrochanteric transverse osteotomy, Crowe type IV DDH, THA, Internal fixation

\section{Background}

Total hip arthroplasty is recommended for patients with developmental dysplasia of the hip who remain disabled after receiving conservative treatment alone [1]. However, THA is a highly difficult surgery in patients with Crowe type IV DDH. Some Crowe type IV DDH patients undergoing THA require a femoral shortening osteotomy to equalize the length of the lower extremities and decrease the difficulty of intraoperative reduction and the incidence of complications [2-4]. The femoral

\footnotetext{
* Correspondence: xuweihua@hust.edu.cn

${ }^{1}$ Department of Orthopaedics, Union Hospital, Tongji Medical College, Huazhong University of Science and Technology, Wuhan 430022, China Full list of author information is available at the end of the article
}

subtrochanteric transverse osteotomy is one of the most common forms of osteotomy [5, 6]. Although a subtrochanteric transverse osteotomy has significant efficacy for the treatment of Crowe type IV DDH patients [4, 7], some complications still occur, including intraoperative osteotomy fracture, neurovascular injury, postoperative osteotomy nonunion, and hip dislocation, $[6,8]$. These complications seriously affect the postoperative rehabilitation of patients. Our study reports the case of a patient with type IV DDH who underwent THA and experienced nonunion after transverse osteotomy. The causes, prevention and treatment of nonunion after transverse osteotomy are discussed based on literature reports. 


\section{Case presentation}

A 62-year-old male patient was admitted to the hospital due to a 50-year history of intermittent pain and limited activity of the right hip that had been aggravated for 1 month. A radiograph of the pelvis showed high dislocation of the right hip (Fig. 1), and the patient was diagnosed with right Crowe type IV DDH. The patient's visual analogue scale (VAS) score was 8 , and his Harris score was 21. A physical examination showed that the right lower extremity was shortened by $5.3 \mathrm{~cm}$, and the patient had a limp, local tenderness of the right hip, and aggravated pain upon internal and external rotation of the hip. The degrees of right hip joint motion were as follows: flexion, $90^{\circ}$; outreach, $12^{\circ}$; adduction, $14^{\circ}$; internal rotation $10^{\circ}$; and external rotation, $5^{\circ}$. The patient reported no other disease history.

The patient was treated with right THA and femoral subtrochanteric shortening transverse osteotomy. The osteotomy was located $1.6 \mathrm{~cm}$ below the lesser trochanter, the length of the osteotomy was $2.7 \mathrm{~cm}$, and steel wires were attached at both ends of the osteotomy to prevent fracture. The intraoperative characteristics of the Johnson company S-ROM prosthesis are as follows: bio-type, $44 \mathrm{~mm}$ acetabular cup, $28 \mathrm{~mm}$ polyethylene liner, $28 \mathrm{~mm}$ ceramic head, and standard shank. The patient was treated postoperatively with antibiotics, analgesics, anticoagulants, and gastroprotectants. A radiograph of both lower extremities and the right hip showed satisfactory positioning of the prosthesis after the operation (Fig. 2). One day after surgery, the patient could walk in the ward with the help of a walker. One week after surgery, the patient could walk freely but limped because of an evidently tilted pelvis. The patient's VAS score was 3 , and his Harris score was 60 . At 40 days after surgery, the patient could put on his socks by himself, and the limp and pelvic tilt were significantly corrected after following the training regimen of the rehabilitation plan. The patient was instructed to practice squatting to facilitate using the toilet. The two ends of osteotomy were in good contact with each other (Fig. 3). The patient's VAS score was 2, and his Harris score was 79 . Three and a half months after the operation, the patient reported a slight sensation of bone rubbing and mild pain in the operated hip. The patient's VAS score was 3, and his Harris score was 70. The ends of the osteotomy had rotated and united poorly (Fig. 4). We recommended that the patient undergo hospitalization to receive internal fixation, but the patient requested to continue observation. Five months after the operation, the patient had obvious sensations of bone rubbing, and his right hip pain was significantly worse. The patient's VAS score was 5 , and his Harris score was 52. The ends of the osteotomy had rotated and exhibited nonunion (Fig. 5). The patient had obvious symptoms of pain, and hospitalization for surgery was recommended.

The patient was diagnosed with nonunion of the osteotomy and was again admitted for plate and screw internal fixation with bone morphogenetic protein (BMP). The patient was treated postoperatively with antibiotics, analgesics, anticoagulants, and gastroprotectants. Three days after surgery, the patient reported a significant reduction in pain, and the sensation of bone rubbing was
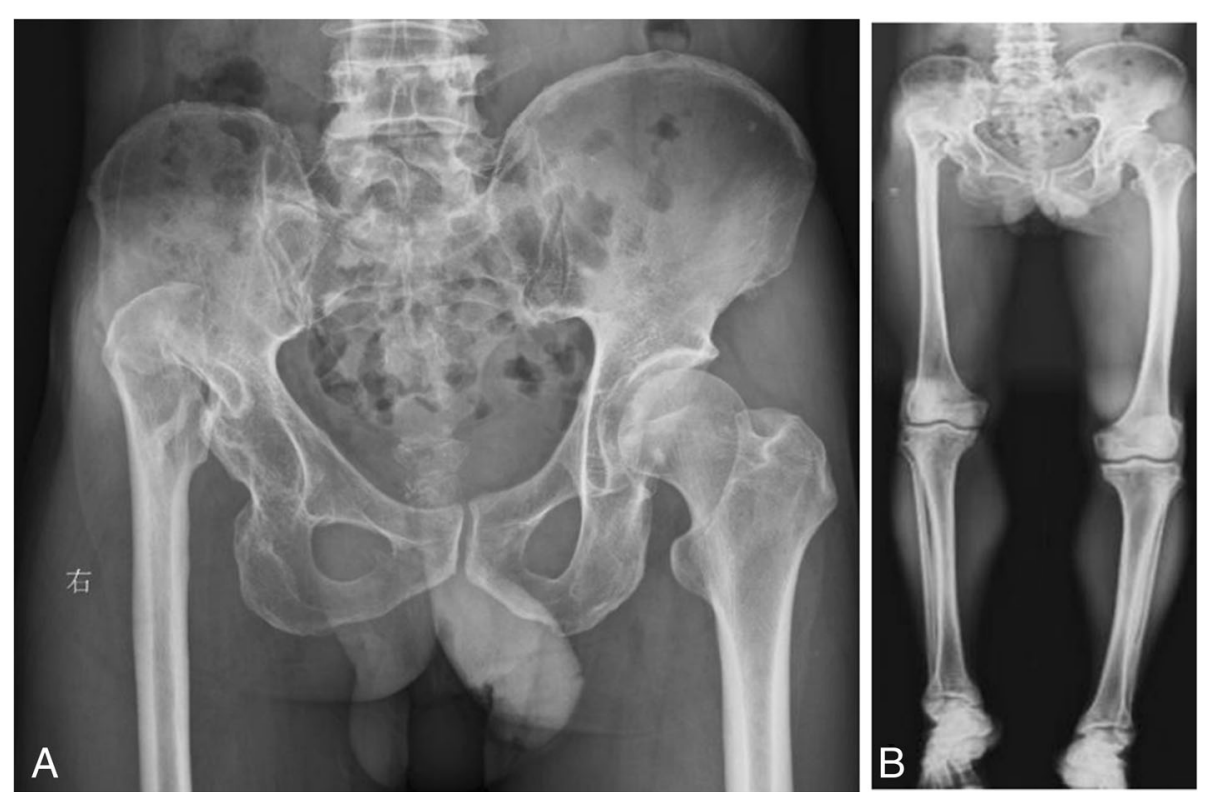

Fig. 1 Radiographs of the pelvis and both lower extremities before the operation. (a) anteroposterior view of the pelvis, (b) anteroposterior view of both lower extremities 

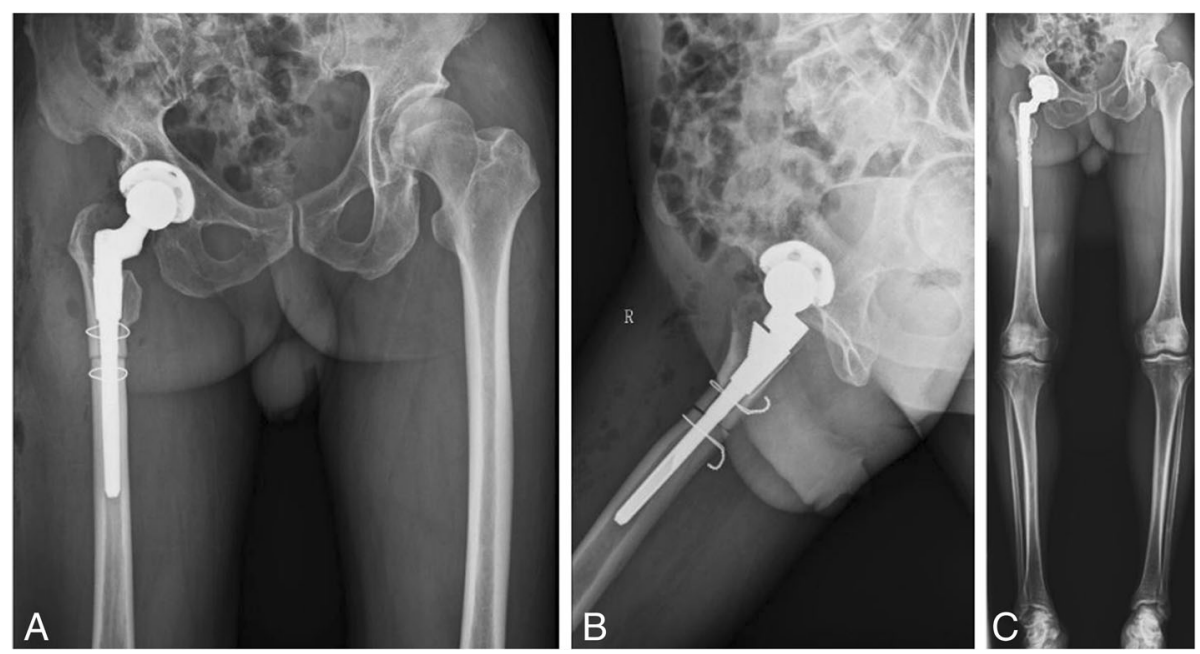

Fig. 2 Radiographs of both lower extremities and the right hip after the operation. (a) anteroposterior view of the right hip, (b) lateral view of the right hip, (c) anteroposterior view of both lower extremities

absent. The patient's VAS score was 3, and his Harris score was 60. A radiograph of the right hip and both lower extremities showed that the plate and screw was well fixed (Fig. 6). One month after internal fixation was applied, the patient reported that his situation was good. The patient's VAS score was 2, and his Harris score was 78. The ends of the osteotomy had not rotated and had begun to exhibit union (Fig. 7). Two and a half months after internal fixation was applied, the patient's VAS score was 2, and his Harris score was 85 . The ends of the osteotomy were firmly fixed, and the union was progressing satisfactorily (Fig. 8). Five months after internal fixation was applied, the patient had only occasional slight pain that did not affect his life. The patient's VAS score was 1 , and his Harris score was 87 . Bone scabs had formed at the ends of the osteotomy (Fig. 9). Eight months after internal fixation was applied, the patient reported no problems with normal activities. His VAS score was 0 , and his Harris score was 90. The ends of the osteotomy had united (Fig. 10).

\section{Discussion}

High dislocation of the femoral head and soft tissue contracture around the hip in patients with Crowe type IV DDH make intraoperative hip joint reduction difficult [8]. Femoral osteotomy can be performed to decrease the difficulty of intraoperative reduction and the incidence rate of complications caused by forced reduction in these patients $[2-4,9,10]$. Although multiple osteotomy methods can be used for patients with Crowe type IV DDH, the femoral subtrochanteric shortening transverse osteotomy is one of the most common techniques $[5,6]$.

Femoral subtrochanteric transverse osteotomy has been clinically shown to have satisfactory efficacy. However, some cases of nonunion still occur. According to

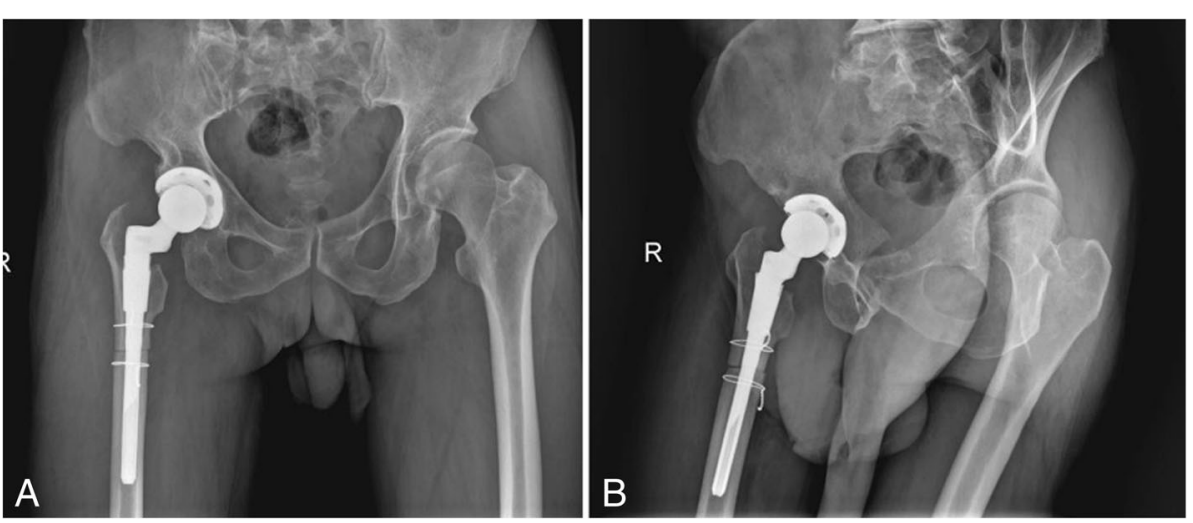

Fig. 3 Radiographs of the right hip at 40 days after the operation. (a) anteroposterior view of the right hip, (b) lateral view of the right hip 


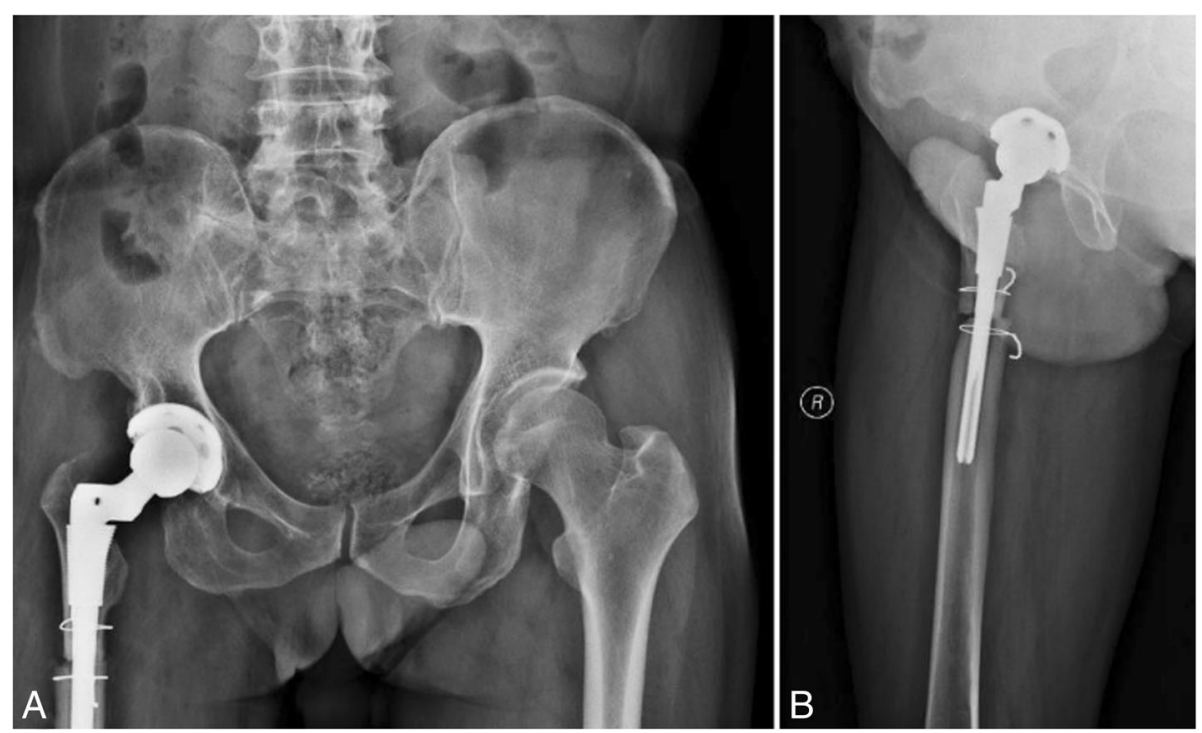

Fig. 4 Radiographs of the right hip at three and a half months after the operation. (a) anteroposterior view of the right hip, (b) lateral view of the right hip

literature reports, the nonunion rate of transverse osteotomy is $1.3 \sim 20 \%$ (Table 1 ) [2-4, 6, 11-17]. The possible reasons for the occurrence of nonunion, as in our case, are as follows. First, excessive periosteal stripping may have reduced or interrupted the blood supply to the osteotomy site, resulting in nonunion of the osteotomy $[2,4,8,13]$. Second, the high local temperature of the saw may have affected the union of the osteotomy site $[4,15]$. Third, the process of reaming can injure the endosteum and cause local necrosis of the bone marrow cavity, which may loosen an originally stable prosthesis and affect union [7, 12, 13]. Fourth, incomplete matching of the round stem and the femoral medullary elliptical cavity may result in rotation and affect union. Fifth, soft tissue embedded in the osteotomy ends may affect union. Sixth, premature load or inappropriate activity may lead to nonunion.

Effective measures should be taken to avoid nonunion in these difficult cases. First, a large and sufficiently long distal femoral stem should be selected to prevent rotation, which can result in nonunion $[6,18]$. Second, the selected femoral prosthesis could be relatively small for patients undergoing surgery of the minor proximal medullary cavity. The distal femoral stem does not have a

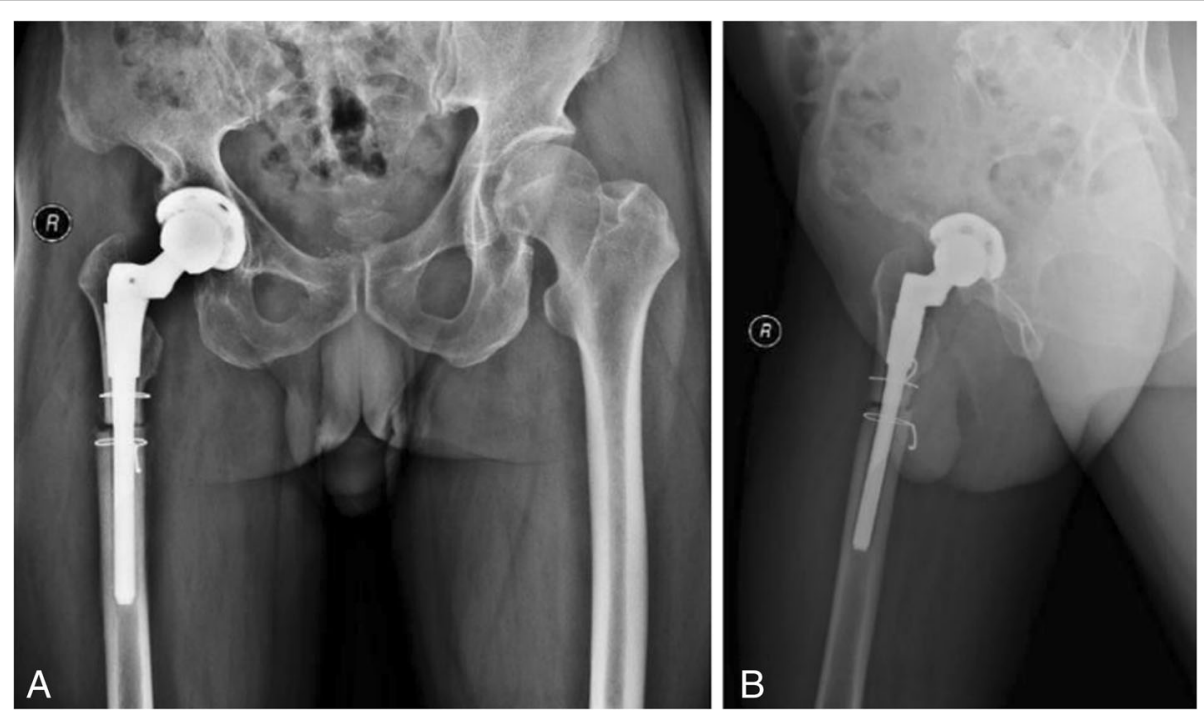

Fig. 5 Radiographs of the right hip at 5 months after the operation. (a) anteroposterior view of the right hip, (b) lateral view of the right hip 


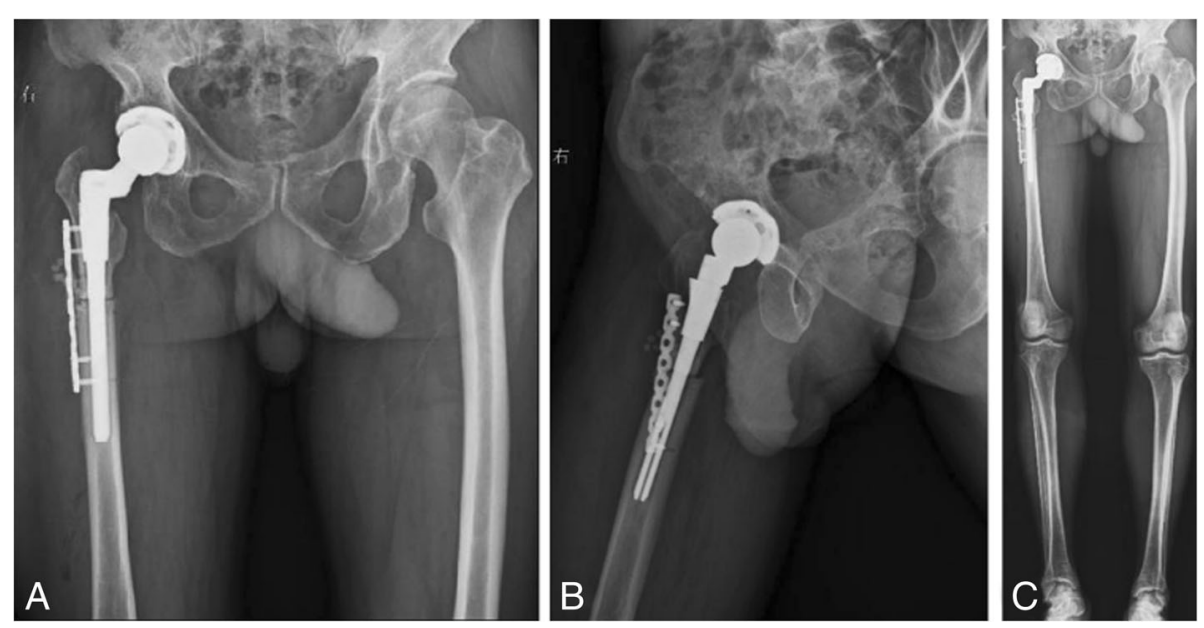

Fig. 6 Radiographs of the right hip and both lower extremities after internal fixation was applied. (a) anteroposterior view of the right hip, (b) lateral view of the right hip, (c) anteroposterior view of both lower extremities

sufficient ability to prevent rotation. Plate and screw internal fixation is a good choice to increase the stability of the osteotomy ends and promote union [19, 20]. Third, the ends of the osteotomy need to be as smooth as possible to increase contact $[4,15]$. Fourth, periosteal stripping should be reduced during the osteotomy procedure [4]. Fifth, application of cold saline flushing is beneficial to reduce damage to bone tissue during osteotomy.The S-ROM femoral prosthesis is applied using the following procedure. The proximal cuff, which has a rough outer surface, is tightly attached to the interior part of the proximal femur. The inner part of the proximal cuff is strongly attached to the tapered femoral stem. The longitudinal streak of the distal portion of the femoral stem is tightly pressed against the medullary cavity. This configuration prevents the S-ROM femoral prosthesis from rotating $[7,18]$. The prosthesis may rotate because the distal femoral stem is too small or the implantation force of the femoral prosthesis is not sufficient. In our case, the S-ROM femoral prosthesis may not have prevented rotation, which impacted the union of the osteotomy ends. Therefore, the medullary cavity of the femur should be fully reamed, and an S-ROM femoral prosthesis that matches the distal femur should be used.

The most common methods of performing subtrochanteric osteotomy include transverse, step-cut, or double chevron techniques $[5,15,16]$. The two most prominent advantages of subtrochanteric transverse osteotomy are simple operation and a freely adjustable anteversion angle. The disadvantage of this osteotomy is that the incidence of nonunion is relatively high compared to that of other of osteotomy types. Subtrochanteric step-cut and double chevron

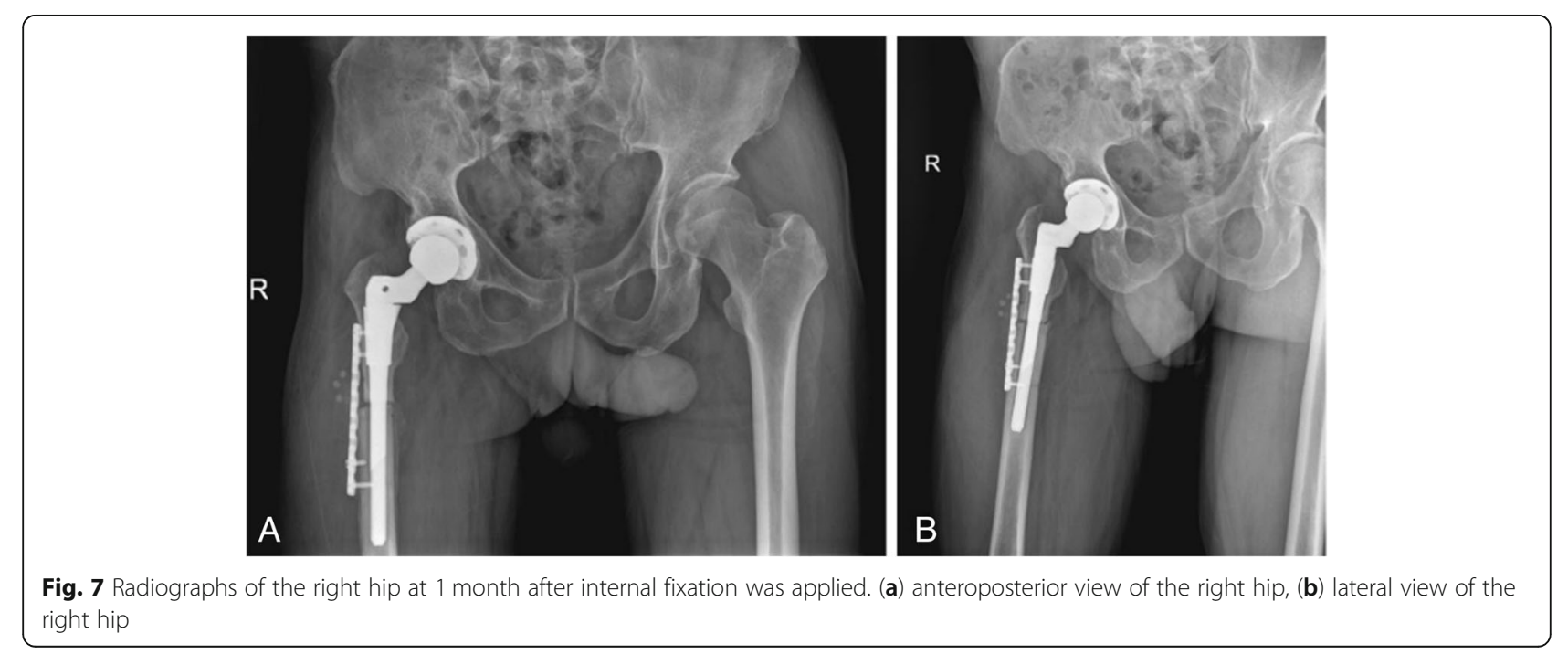




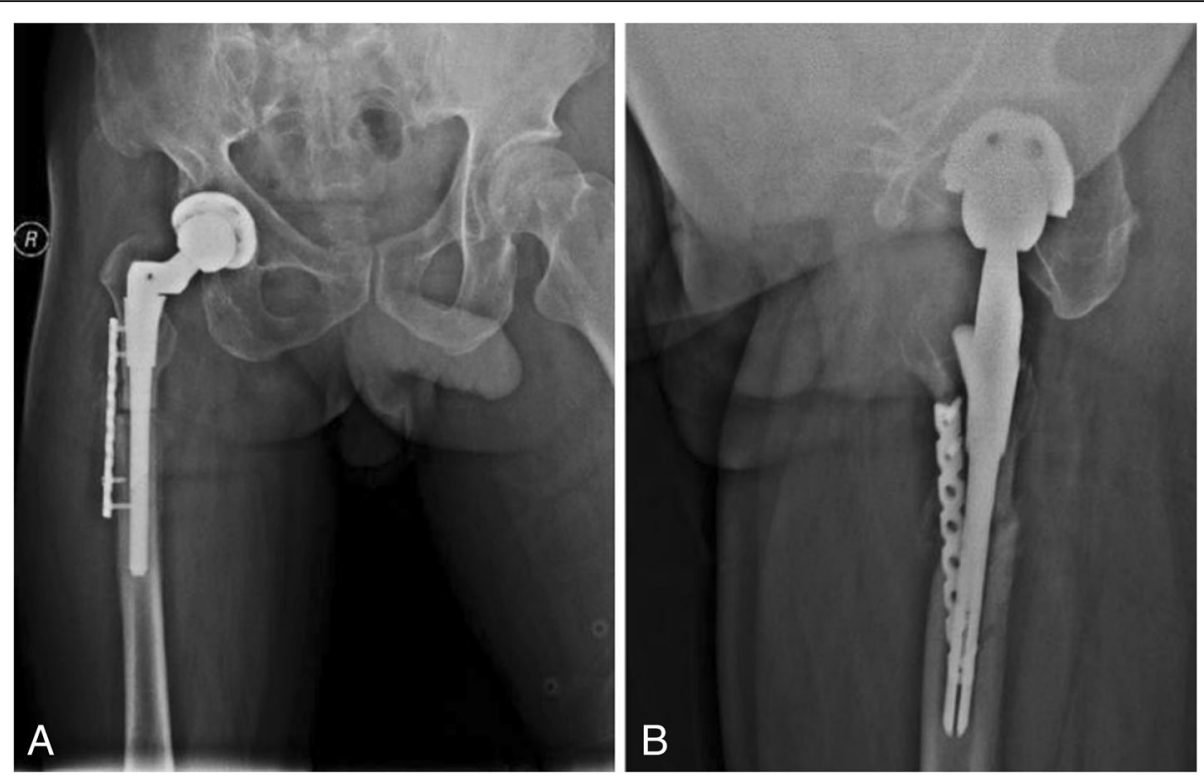

Fig. 8 Radiographs of the right hip at two and a half months after internal fixation was applied. (a) anteroposterior view of the right hip, (b) lateral view of the right hip

osteotomies have a relatively low incidence of nonunion, but these two osteotomy methods have higher technical requirements. The major disadvantage of these methods is that adjustment of anteversion is difficult if the osteotomy fails. In our case, rotational instability of the transverse osteotomy led to nonunion.

The following improvements will be implemented for similar cases in the future. The size and type of the femoral medullary cavity should be carefully evaluated

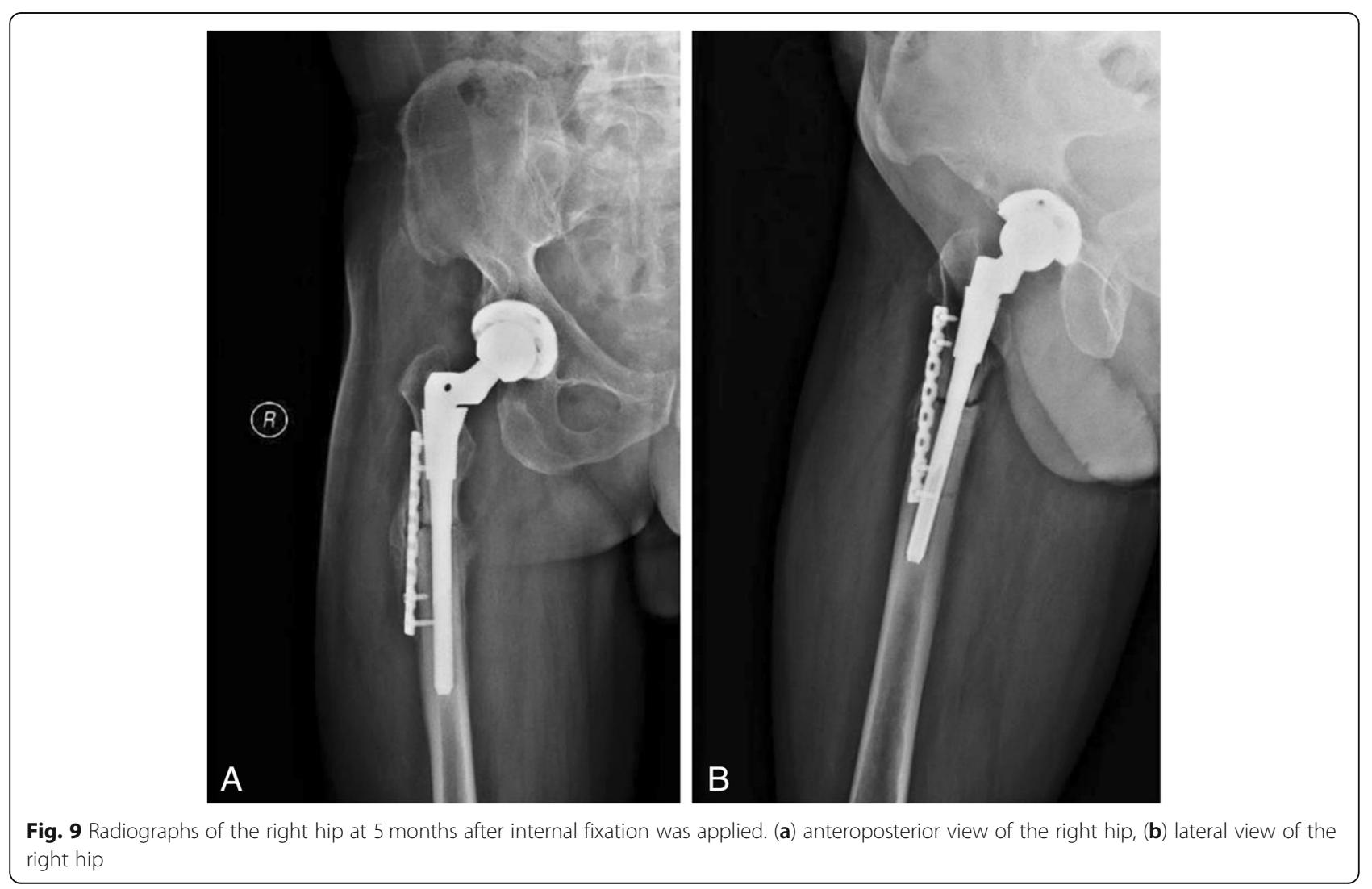



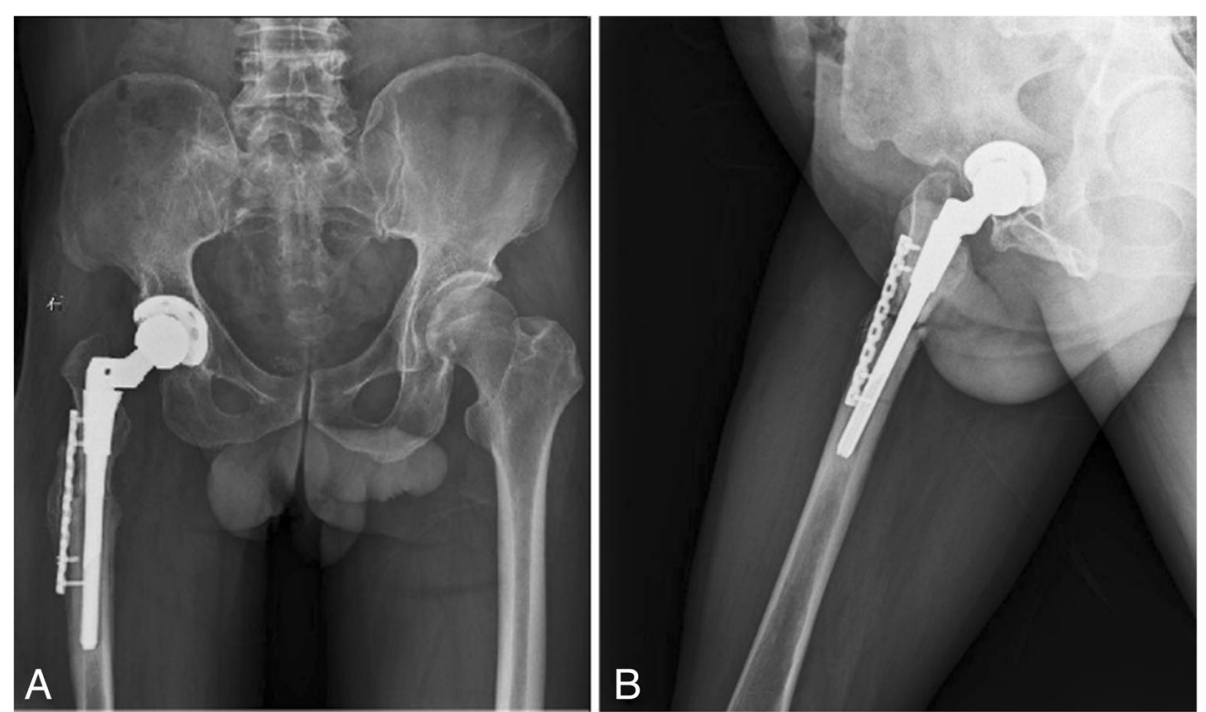

Fig. 10 Radiographs of the right hip at 8 months after internal fixation was applied. (a) anteroposterior view of the right hip, (b) lateral view of the right hip

preoperatively. The femoral medullary cavity should be sufficiently reamed intraoperatively. A long and sufficiently large femoral prosthesis should be selected to prevent rotation. We currently use a cortical bone plate with a steel cable or plate and screw internal fixation to increase the stability of the osteotomy ends. We will continue to perform and utilize the advantages of subtrochanteric transverse osteotomy while taking effective measures to avoid its associated complications.

\section{Conclusions}

THA with femoral subtrochanteric transverse osteotomy can provide good clinical efficacy and result in a relatively high rate of union in Crowe type IV DDH patients. However, some patients still experience nonunion. The causes of nonunion should be carefully analysed, and measures should be actively taken to prevent its

Table 1 Summary of the literature on osteotomy nonunion

\begin{tabular}{llll}
\hline Study & Total & Nonunion & Percentage \\
\hline Akiyama et al. [11] & 15 & 3 & $20 \%$ \\
Charity et al. [12] & 18 & 1 & $5.6 \%$ \\
Wang et al. [13] & 56 & 2 & $3.6 \%$ \\
Sofu et al. [14] & 73 & 4 & $5.5 \%$ \\
Wang et al. [4] & 76 & 1 & $1.3 \%$ \\
Mu et al. [15] & 71 & 2 & $2.8 \%$ \\
Ollivier et al. [3] & 28 & 2 & $7.1 \%$ \\
Krych et al. [2] & 28 & 2 & $7.1 \%$ \\
Masonis et al. [16] & 24 & 2 & $8.3 \%$ \\
Park et al. [6] & 24 & 3 & $12.5 \%$ \\
Yalcin et al. [17] & 44 & 5 & $8.8 \%$ \\
\hline
\end{tabular}

occurrence. Plate and screw internal fixation with BMP is an effective treatment choice if nonunion occurs.

\section{Abbreviations \\ BMP: Bone morphogenetic protein; DDH: Developmental dysplasia of the hip; THA: Total hip arthroplasty; VAS: Visual analogue scale}

\section{Acknowledgements}

No one else contributed to the article except for the authors.

\section{Funding}

This work was supported by a grant from the National Natural Science Foundation of China (No. 81672155).

\section{Availability of data and materials}

All the data supporting our findings are contained within the manuscript.

\section{Authors' contributions}

SG made substantive intellectual contributions to the study and wrote the paper. WHX strictly verified the authenticity and professionalism of the article. RYW and LZH evaluated the VAS scores during the follow-up period. SKL and GC contributed to the imaging evaluations. BW helped perform the literature search. All authors read and approved the final manuscript.

\section{Ethics approval and consent to participate} Not applicable.

\section{Consent for publication}

Informed written consent was obtained from the patient to publish the case-related information.

\section{Competing interests}

All authors declare that they have no competing interests.

\section{Publisher's Note}

Springer Nature remains neutral with regard to jurisdictional claims in published maps and institutional affiliations.

\section{Author details}

${ }^{1}$ Department of Orthopaedics, Union Hospital, Tongji Medical College, Huazhong University of Science and Technology, Wuhan 430022, China. 
${ }^{2}$ Department of Rehabilitation, Union Hospital, Tongji Medical College, Huazhong University of Science and Technology, Wuhan 430022, China.

Received: 18 September 2018 Accepted: 3 May 2019

Published online: 10 May 2019

\section{References}

1. Yang S, Cui Q. Total hip arthroplasty in developmental dysplasia of the hip: Review of anatomy, techniques and outcomes. World J Orthop. 2012;3(5):42-8.

2. Krych AJ, Howard JL, Trousdale RT, Cabanela ME, Berry DJ. Total hip arthroplasty with shortening subtrochanteric osteotomy in Crowe type-IV developmental dysplasia. J Bone Joint Surg Am. 2009;91(9):2213-21.

3. Ollivier M, Abdel MP, Krych AJ, Trousdale RT, Berry DJ. Long-Term Results of Total Hip Arthroplasty with Shortening Subtrochanteric Osteotomy in Crowe IV Developmental Dysplasia. J Arthroplast. 2016:31(8):1756-60.

4. Wang D, Li LL, Wang HY, Pei FX, Zhou ZK. Long-Term Results of Cementless Total Hip Arthroplasty with Subtrochanteric Shortening Osteotomy in Crowe Type IV Developmental Dysplasia. J Arthroplast. 2017;32(4):1211-9.

5. Muratli KS, Karatosun V, Uzun B, Celik S. Subtrochanteric shortening in total hip arthroplasty: biomechanical comparison of four techniques. J Arthroplast. 2014;29(4):836-42.

6. Park MS, Kim KH, Jeong WC. Transverse subtrochanteric shortening osteotomy in primary total hip arthroplasty for patients with severe hip developmental dysplasia. J Arthroplast. 2007;22(7):1031-6.

7. Zeng WN, Liu JL, Wang FY, Zhang X, Fan HQ, Chen GX, Guo L, Duan XJ, Zhou Q, Yang L. Total hip arthroplasty for patients with Crowe type IV developmental dysplasia of the hip: Ten years results. Int J Surg. 2017;42:17-21.

8. Greber EM, Pelt CE, Gililland JM, Anderson MB, Erickson JA, Peters CL. Challenges in Total Hip Arthroplasty in the Setting of Developmental Dysplasia of the Hip. J Arthroplast. 2017;32(9S):S38-44.

9. Hua WB, Yang SH, Xu WH, Ye SN, Liu XZ, Wang J, Feng Y. Total hip arthroplasty with subtrochanteric femoral shortening osteotomy for high hip dislocation. Orthop Surg. 2015;7(2):112-8.

10. Sonohata M, Kitajima M, Kawano S, Mawatari M. Nerve Palsy after Total Hip Arthroplasty without Subtrochanteric Femoral Shortening Osteotomy for a Completely Dislocated Hip Joint. Open Orthop J. 2016;10:785-92.

11. Akiyama H, Kawanabe K, Yamamoto K, Kuroda Y, So K, Goto K, Nakamura T. Cemented total hip arthroplasty with subtrochanteric femoral shortening transverse osteotomy for severely dislocated hips: outcome with a 3- to 10year follow-up period. J Orthop Sci. 2011;16(3):270-7.

12. Charity JA, Tsiridis E, Sheeraz A, Howell JR, Hubble MJ, Timperley AJ, Gie GA Treatment of Crowe IV high hip dysplasia with total hip replacement using the Exeter stem and shortening derotational subtrochanteric osteotomy. J Bone Joint Surg Br. 2011:93(1):34-8.

13. Wang D, Li DH, Li Q, Wang HY, Luo ZY, Yang Y, Pei FX, Zhou ZK. Subtrochanteric shortening osteotomy during cementless total hip arthroplasty in young patients with severe developmental dysplasia of the hip. BMC Musculoskelet Disord. 2017;18(1):491.

14. Sofu H, Kockara N, Gursu S, Issin A, Oner A, Sahin V. Transverse Subtrochanteric Shortening Osteotomy During Cementless Total Hip Arthroplasty in Crowe Type-III or IV Developmental Dysplasia. J Arthroplast. 2015;30(6):1019-23.

15. Mu W, Yang D, Xu B, Mamtimin A, Guo W, Cao L. Midterm Outcome of Cementless Total Hip Arthroplasty in Crowe IV-Hartofilakidis Type III Developmental Dysplasia of the Hip. J Arthroplast. 2016;31(3):668-75.

16. Masonis JL, Patel JV, Miu A, Bourne RB, McCalden R, Macdonald SJ, Rorabeck $\mathrm{CH}$. Subtrochanteric shortening and derotational osteotomy in primary total hip arthroplasty for patients with severe hip dysplasia: 5-year follow-up. J Arthroplast. 2003:18(3 Suppl 1):68-73.

17. Yalcin N, Kilicarslan K, Karatas F, Mutlu T, Yildirim H. Cementless total hip arthroplasty with subtrochanteric transverse shortening osteotomy for severely dysplastic or dislocated hips. Hip Int. 2010;20(1):87-93.

18. Onodera S, Majima T, Ito H, Matsuno T, Kishimoto T, Minami A. Cementless total hip arthroplasty using the modular S-ROM prosthesis combined with corrective proximal femoral osteotomy. J Arthroplast. 2006;21(5):664-9.

19. Yasgur DJ, Stuchin SA, Adler EM, DiCesare PE. Subtrochanteric femoral shortening osteotomy in total hip arthroplasty for high-riding developmental dislocation of the hip. J Arthroplast. 1997;12:880

20. Bruce WJ, Rizkallah SM, Kwon YM, Goldberg JA, Walsh WR. A new technique of subtrochanteric shortening in total hip arthroplasty: surgical technique and results of 9 cases. J Arthroplast. 2000;15:617.

Ready to submit your research? Choose BMC and benefit from:

- fast, convenient online submission

- thorough peer review by experienced researchers in your field

- rapid publication on acceptance

- support for research data, including large and complex data types

- gold Open Access which fosters wider collaboration and increased citations

- maximum visibility for your research: over $100 \mathrm{M}$ website views per year

At BMC, research is always in progress.

Learn more biomedcentral.com/submissions 